\title{
Particle Identification in the LHCb Experiment
}

\author{
F.J.P. Soler ${ }^{1,2, *}$ \\ 1 University of Glasgow, Glasgow, United Kingdom \\ e-mail: p.soler@physics.gla.ac.uk \\ 2 Rutherford Appleton Laboratory, Chilton, Didcot, Oxfordshire, United Kingdom \\ e-mail: p.soler@rl.ac.uk
}

\begin{abstract}
The present article describes the ring imaging Cherenkov (RICH) detectors of the $\mathrm{LHCb}$ experiment. The two RICH detectors of $\mathrm{LHCb}$ contain three radiators, carefully designed to provide particle identification of B meson decays in the relevant momentum range of the experiment. A status of the design of the RICH system will be presented with the expected overall performance.
\end{abstract}

PACS: $13.20 . \mathrm{Hw} ; 14.40 . \mathrm{Nd} ; 29.40 . \mathrm{Ka}$

\section{Introduction}

The LHCb experiment is an experiment that is being built at the Large Hadron Collider (LHC) to test our understanding of $\mathrm{CP}$ violation by measuring the decays of $\mathrm{B}$ mesons in a variety of different channels. The LHCb experiment is designed to exploit the unique features of the LHC in terms of energy and production rate, allowing unprecedented accuracy in the measurement of the parameters of the unitarity triangles derived from the Cabibbo-Kobayashi-Maskawa (CKM) quark mixing matrix. A comprehensive review of the status and physics to be carried out in LHCb is included in the current conference proceedings [1].

One of the crucial requirements of LHCb is particle identification. This enables us to distinguish between pions and kaons in a variety of $\mathrm{B}$ meson final state decays, needed to separate $\mathrm{CP}$ violating channels from background. Particle identification in LHCb is achieved using ring-imaging Cherenkov (RICH) detectors [2]. This paper will briefly describe the RICH system of LHCb. We will identify the physics requirements of the experiment and present a general overview of the system. Then we will concentrate in the details of the photon detector technology and a description of the readout electronics. The paper ends with the overall performance of the LHCb RICH, including two selected channels of interest.

\section{Particle identification requirements and $\mathrm{RICH}$ detector overview}

$\mathrm{LHCb}$ is a forward single arm spectrometer with an acceptance of 10-300 mrad in the bending (horizontal) plane of the LHCb dipole magnet and 10-250 mrad

* On Behalf of the LHCb collaboration. 


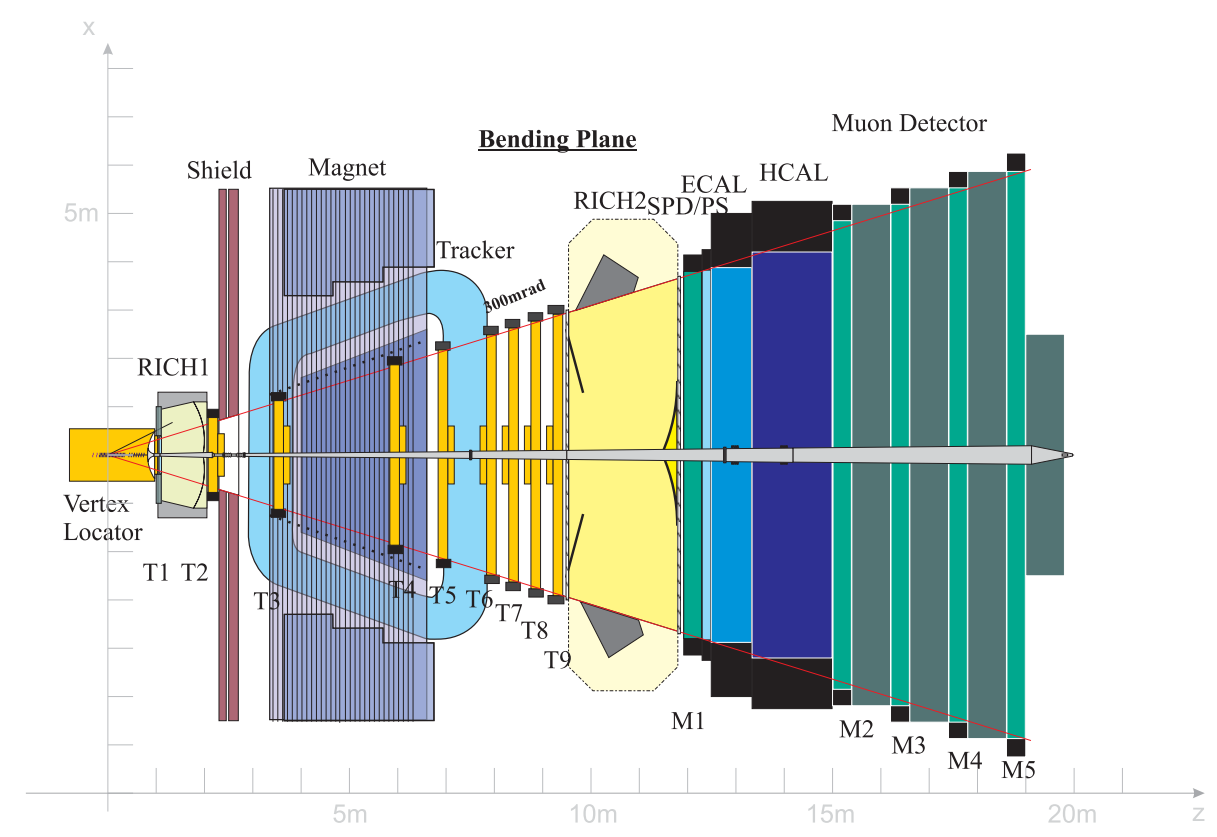

Fig. 1. LHCb spectrometer seen from above (cut in the bending plane), showing the location of RICH-1 and RICH-2 detectors.

in the non-bending (vertical) plane (see Fig. 1). A measurement of the CP asymmetry of $B_{d}^{0} \rightarrow \pi^{+} \pi^{-}$decays will provide information about the angle $\alpha$ of the unitarity triangle, making it necessary to distinguish these decays from two body backgrounds $\left(B_{d}^{0} \rightarrow K^{+} \pi^{-}, B_{d}^{0} \rightarrow K^{-} \pi^{+}, B_{s}^{0} \rightarrow K^{+} K^{-}\right)$. Since $90 \%$ of decays have momentum $p<150 \mathrm{GeV} / \mathrm{c}$, we need hadron identification to the highest possible momentum. In addition, the identification of kaons from the accompanying $b$ hadron decay in the event provides a powerful flavour tag, with the charge of the kaon determining the charge of the initial b quark. Kaons from high multiplicity decays can have momenta as low as $1 \mathrm{GeV} / \mathrm{c}$, so this sets the lower momentum limit for particle identification.

The requirement of particle identification in a momentum range from 1 to $150 \mathrm{GeV} / \mathrm{c}$ imposes the use of a ring-imaging Cherenkov (RICH) system. Fig. 2 shows the correlation of momentum with track angle for simulated $B_{d}^{0} \rightarrow \pi^{+} \pi^{-}$ decays. The strategy adopted by $\mathrm{LHCb}$ is to have three radiators with different refractive indices (silica aerogel for low momentum tracks, gaseous $C_{4} F_{10}$ for intermediate momentum and $C F_{4}$ gas for high momentum tracks) within two separate RICH detectors (named RICH-1 and RICH-2) to cover different acceptance requirements.

Fig. 1 shows a schematic diagram of the LHCb spectrometer with the RICH detectors. RICH-1 is placed close to the vertex detector, covering an acceptance of $300 \mathrm{mrad}$ horizontally ( $250 \mathrm{mrad}$ vertically) and containing the aerogel and $C_{4} F_{10}$ radiators. The radiator volume has dimensions $2.4 \times 2.4 \times 1 \mathrm{~m}^{3}$ and 


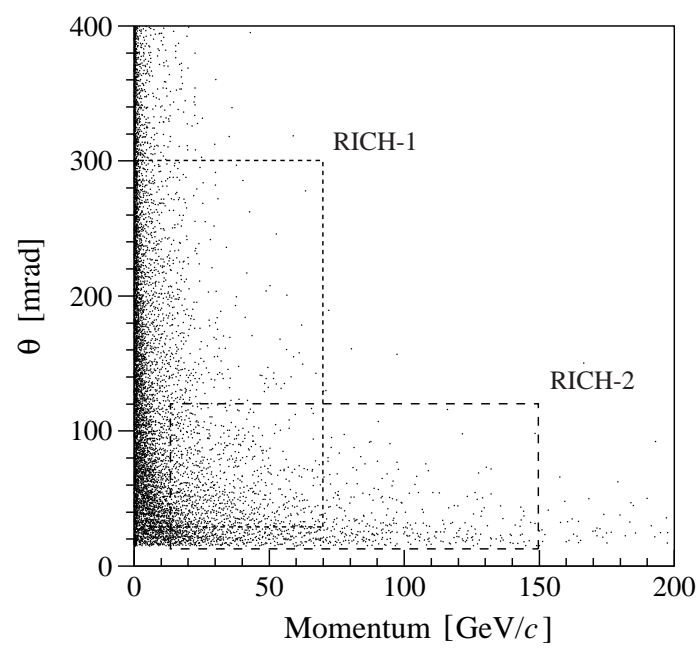

Fig. 2. Polar angle $\theta$ versus momentum for $B_{d}^{0} \rightarrow \pi^{+} \pi^{-}$decays, with the RICH-1 and $\mathrm{RICH}-2$ regions of interest indicated by dashed lines.

contains a set of spherical mirrors (radius of curvature $1.70 \mathrm{~m}$ ) to focus the Cherenkov light on the photon detector plane. The overall radiation length of the current design, which inludes $5 \mathrm{~cm}$ aerogel and glass mirrors supported by a carbon-fibre three-point mount is $14 \% X_{0}$. Efforts are underway to reduce this material budget by manufacturing the mirrors either out of beryllium or composite materials (with about $1 \% X_{0}$ instead of $4.5 \% X_{0}$ for glass).

The most transparent aerogel available for the RICH-1 detector is hygroscopic aerogel of thickness $5 \mathrm{~cm}$ manufactured in Novosibirsk. Rayleigh scattering of light limits the useful wavelength range of the Cherenkov light from the aerogel. The transmission through a length $\mathrm{L}$ is proportional to $e^{-C L / \lambda^{4}}$, with $\mathrm{C}$ being the clarity coefficient, which was measured at a test beam to be $C=0.0045 \mu \mathrm{m}^{4} / \mathrm{cm}^{-1}$ with a refractive index of 1.034 . This aerogel is radiation hard, without any significant loss of transmission after a dose of $10^{4}$ Gy (approximately 1 year of running with $\mathrm{LHCb}$ ).

RICH-2, placed further downstream, is designed to identify high momentum, low angle tracks (acceptance less than $120 \mathrm{mrad}$ horizontally and $100 \mathrm{mrad}$ vertically) and contains the $C F_{4}$ radiator. The gas volume dimensions are $7 \times$ $7 \times 2.45 \mathrm{~m}^{3}$ and the detector contains two sets of mirrors for focusing the light on the photon detector plane (a set of spherical mirrors of radius $8.70 \mathrm{~m}$ and a set of flat mirrors). The gas enclosure is made up of two low mass windows, with about $30 \mathrm{~mm}$ of a light polymethacrylimid (PMI) foam with $1 \mathrm{~mm}$ thick carbon fibre skins in the upstream window and $1 \mathrm{~mm}$ aluminium skins in the downstream window. The total radiation thickness is $12.4 \% X_{0}$. A tube of $2 \mathrm{~mm}$ thick carbon fibre surrounds the beam pipe. The photon detector windows are $1500 \times 750 \times 5 \mathrm{~mm}^{3}$ in size and will be made out of two plates of fused silica 


\begin{tabular}{lccc}
\hline \hline Radiator & Aerogel & $C_{4} F_{10}$ & $C F_{4}$ \\
\hline$n$ & 1.03 & 1.0014 & 1.0005 \\
$\theta_{c}^{\max }[\mathrm{mrad}]$ & 242 & 53 & 32 \\
$\sigma_{\theta}[\mathrm{mrad}]$ & 2.0 & 1.45 & 0.58 \\
$N_{\text {pe }}$ & 7 & 33 & 18 \\
$p_{\text {thresh }}(\pi)[\mathrm{GeV} / \mathrm{c}]$ & 0.6 & 2.6 & 4.4 \\
$p_{\text {thresh }}(K)[\mathrm{GeV} / \mathrm{c}]$ & 2.0 & 9.3 & 15.6 \\
\hline \hline
\end{tabular}

Table 1. Characteristics of the radiator materials of the LHCb RICH systems.

glass with a transmission of more than $90 \%$ for wavelengths above $200 \mathrm{~nm}$.

The Cherenkov characteristics of the three radiators are shown in table 1 , with details of the refractive index $(n)$, maximum Cherenkov angle for saturated tracks $\left(\theta_{c}^{\max }\right)$, simulated Cherenkov angle resolution $\left(\sigma_{\theta}\right)$, simulated number of photoelectrons per ring $\left(N_{p e}\right)$ and the threshold momenta for pions and kaons $\left(p_{\text {thresh }}(\pi)\right.$ and $\left.p_{\text {thresh }}(K)\right)$. A careful alignment of the RICH detectors will be required to achieve the stated Cherenkov angle resolutions. Alignment parameters can be determined by reconstructing saturated Cherenkov rings. The alignment will have a negligible influence in the Cherenkov angle resolution if the initial seed alignment data is known to an accuracy of $0.5 \mathrm{mrad}$ in the tilt angles of the RICH mirrors [3].

\section{Photon detectors and electronics}

The photon detectors have very demanding requirements. They need to cover a photon detector area of $2.6 \mathrm{~m}^{2}$ providing single photon sensitivity between 200 and $600 \mathrm{~nm}$ and with a quantum efficiency greater than $20 \%$ in this wavelength range and a large active area fraction of about $70 \%$. To be able to achieve the Cherenkov angle resolution required, we also need a granularity of approximately $2.5 \times 2.5 \mathrm{~mm}^{2}$. In addition, the read-out electronics of these photon detectors need to operate at LHC speeds of $40 \mathrm{MHz}$ and they need to operate in an environment with stray magnetic fields and charged particle backgrounds.

The photon detector of choice is the pixel hybrid photodiode (HPD), prototypes of which have been manufactured by the Dutch firm DEP (Delft Electronische Producten). Fig. 3 shows a schematic design for this detector. Each HPD anode consists of a silicon pixel sensor array of 1024 channels $(32 \times 32)$ of size $500 \times 500 \mu \mathrm{m}^{2}$ bump bonded to a binary electronics read-out chip. The photocathode is made out of S20 (multialkali) material deposited on a $7 \mathrm{~mm}$ thick quartz window, with integrated quantum efficiency of $0.77 \mathrm{eV}$. Cross-focusing electronics provide a demagnification factor of 5 from the photocathode to the Si sensor with a point spread function of less than $50 \mu \mathrm{m}$, thereby providing the required $2.5 \times 2.5 \mathrm{~mm}^{2}$ granularity. The effective photocathode diameter is $75 \mathrm{~mm}$, which, with the $87 \mathrm{~mm}$ distance between HPDs in a close packing assembly, provides a photocathode coverage of $67 \%$. The HPDs operate at $-20 \mathrm{kV}$, 
to achieve the required acceleration of the photoelectrons onto the Si sensor and are surrounded by a mu-metal shield. Prototype HPDs have been extensively studied in test beams $[4,5]$, demonstrating the performance of these devices as expected from simulations. Extensive magnetic field tests have been performed of a prototype HPD read out by a CCD with $150 \mu \mathrm{m}$ resolution, showing that the HPD assembly can tolerate both axial and transverse fields up to 10 Gauss but would experience deterioration in the performance for fields larger than this.

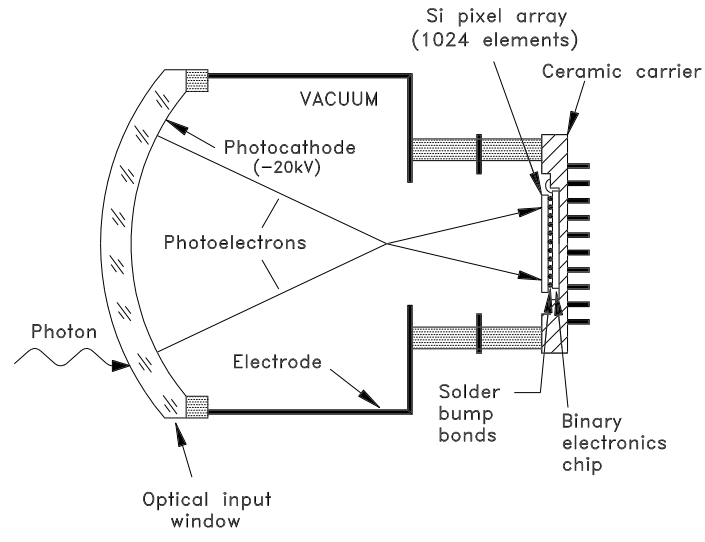

Fig. 3. Schematic design of the hybrid photodiode for the LHCb RICH.

The HPD read-out electronics consists of a 1024 channel, $0.25 \mu \mathrm{m}$ deep submicron radiation tolerant, CMOS, front-end chip with a pitch of $500 \times 500 \mu \mathrm{m}^{2}$ bump bonded to the $\mathrm{Si}$ sensor, operating at $40 \mathrm{MHz}[6,7]$. This development is a combined ALICE/LHCb project. Good quality bump bonding has been provided in industry (VTT, Finland). Preliminary results of the first assemblies show thresholds at $\sim 700$ electrons and noise levels of $\sim 90$ electrons, which comfortably meet the $\mathrm{LHCb}$ requirements of less than 2000 electrons and less than 250 electrons, respectively for the threshold and noise, given a signal of $\sim$ 5000 electrons. However, initial chip prototypes have been found to only operate at $10 \mathrm{MHz}$ and need to be redesigned to achieve the stated aim of operating at $40 \mathrm{MHz}$.

The signals from the pixel chip at $40 \mathrm{MHz}$ will provide Level-0 binary signals with $4 \mu$ s latency in 32 parallel channels read out at $1 \mathrm{MHz}$ into the on-detector Level-0 adapter module (two HPDs per module). A second level of multiplexing $(\times 16)$ is carried out and the data is read through 860 optical links into the off-detector Level-1 electronics at $100 \mathrm{~m}$ distance outside the high radiation area. The Level-1 module buffers the data during the Level-1 latency, removes Level-0 rejected events and transports the data towards the data acquisition event builder network after zero suppression. There are also links to the Timing Trigger and Control (TTC) system and the Detector Control System (DCS). A schematic for the whole electronics is shown in Fig 4.

These electronic modules will also be used for HPD testing facilities to carry 
out the quality control and testing of the 450 HPDs that will be needed to cover the photo-detector area of both RICH detectors.

A potential backup solution that would also meet our requirements as a photon detector for the $\mathrm{LHCb} \mathrm{RICH}$ is the use of multi-anode photomultipier tubes (PMT) fitted with focusing quartz lenses $[5,8]$.

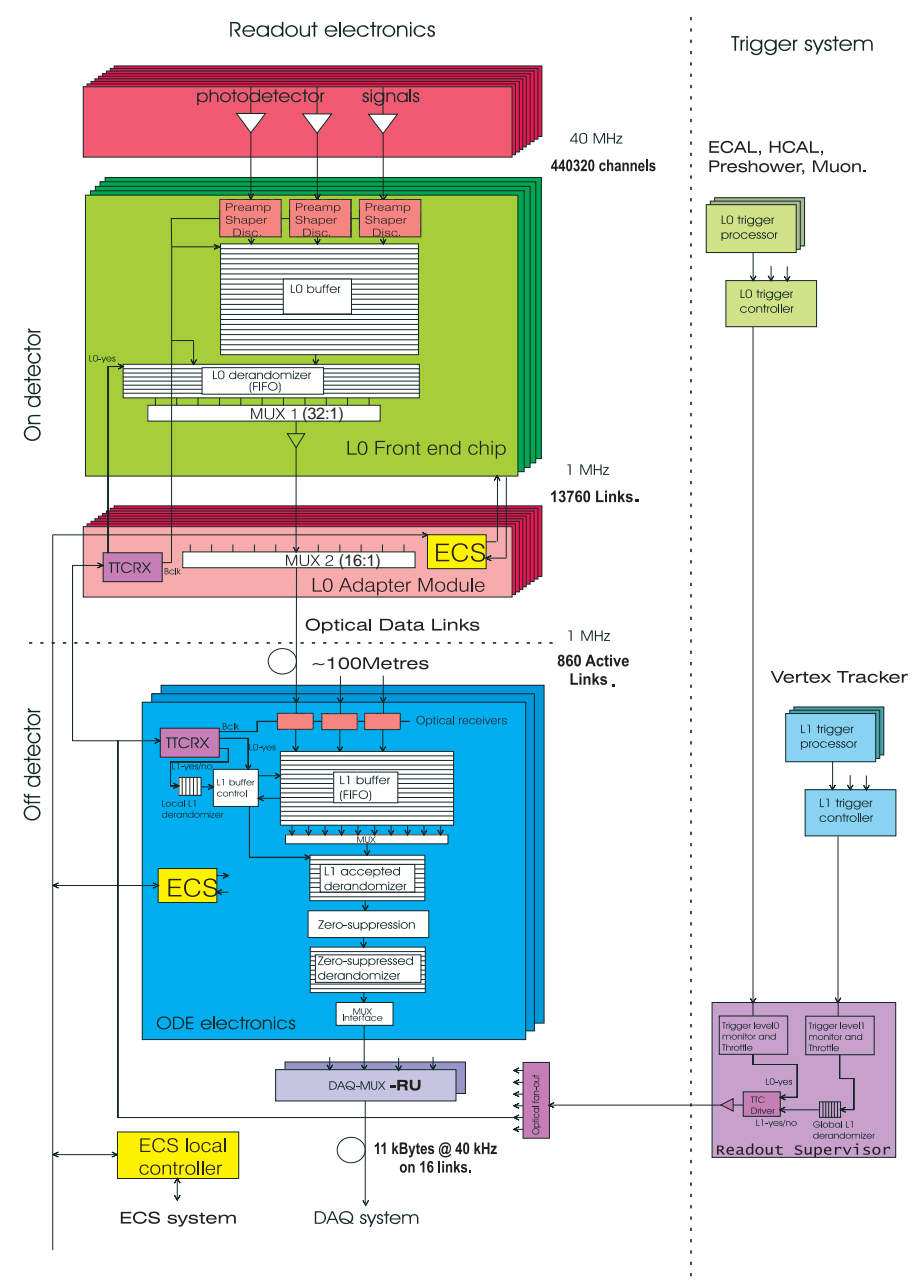

Fig. 4. A schematic of the LHCb RICH readout architecture.

\section{RICH performance and conclusions}

The overall performance of the RICH detectors of LHCb has been calculated through simulations based on the measured test beam HPD data, taking into account a global pattern recognition algorithm for the reconstruction of the 
Cherenkov rings and taking into account background photons from the most up-to-date material description of the detector. Results from the simulation on angular resolution and number of detected photons are summarised in Table 1.

Fig 5 shows the pion-kaon separation capabilities of the combined LHCb RICH system. One obtains more than $3 \sigma$ separation in a range approximately between 3 and $76 \mathrm{GeV} / \mathrm{c}$. In the case of the $B_{d}^{0} \rightarrow \pi^{+} \pi^{-}$decay, sensitive to the angle $\alpha$ of the unitarity triangle, the background rejection capabilities of the RICH (see Fig 6) allow the signal to stand out above background and will allow LHCb to determine $\alpha$ with an accuracy of $2^{\circ}$ to $5^{\circ}$ (depending on the ratio of tree to penguin diagrams) within 1 year. Another example in Fig. 6 is the decay $B_{s} \rightarrow D_{s}^{\mp} K^{ \pm}$, where the RICH can successfully reject $B_{s} \rightarrow D_{s} \pi$ backgrounds and make a measurement of $\gamma-2 \delta \gamma$ with an accuracy between $6^{\circ}$ and $14^{\circ}$.

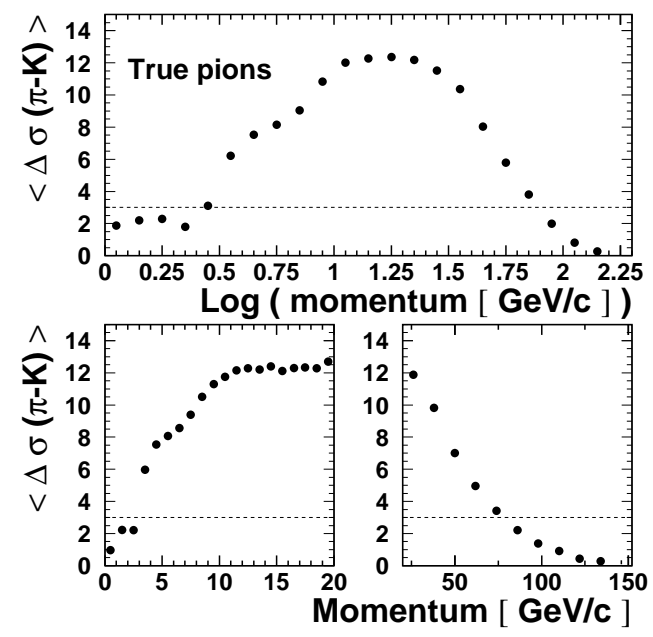

Fig. 5. Pion-kaon separation performance of the LHCb RICH system in terms of number of sigma difference $(\Delta \sigma)$.

The two RICH detectors of LHCb are essential for the LHCb physics programme and the detector design is progressing since the submission of the RICH TDR in October 2000. Hybrid photodiodes are the baseline technology for the photon detectors of LHCb RICH. Despite not being able to operate at $40 \mathrm{MHz}$ yet (a redesign is currently in progress), the initial prototypes for the pixel HPD chip show very good noise and threshold performances well within the LHCb RICH specifications. The design for all subsystems are detailed and advanced and we are in the transition from the $R \& D$ to the construction phase of the project with all indications that the RICH detectors will be in time to take data when LHC becomes operational in 2006. 

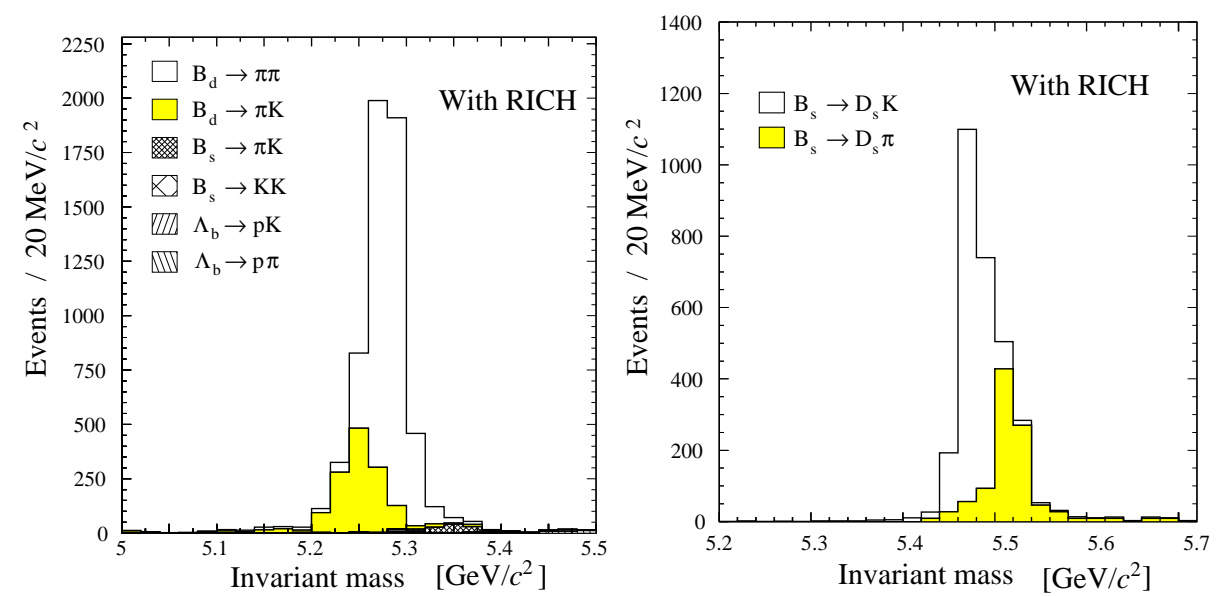

Fig. 6. $B_{d}^{0} \rightarrow \pi^{+} \pi^{-}$signal (left) and $B_{s} \rightarrow D_{s}^{\mp} K^{ \pm}$signal (right) using the RICH detector to suppress background.

\section{Acknowledgements}

The RICH systems of $\mathrm{LHCb}$ are a responsibility of the following institutions within LHCb: CERN, INFN and the Universities of Milano and Genova, the University of Bristol, the University of Cambridge, the University of Edinburgh, the University of Glasgow, Imperial College of Science and Technology (London), the University of Oxford and the Rutherford Appleton Laboratory. We wish to acknowledge the financial contribution of INFN (Italy), CERN and PPARC (U.K.) in the RICH project of LHCb.

\section{References}

1. T. Nakada, "Status of the LHCb Experiment", Proceedings of the III International Symposium on LHC Detectors and Physics (Chia, Sardinia, 2001), Submitted to EPJ C Direct.

2. "LHCb RICH Technical Design Report", CERN-LHCC 2000-037 (September 2000).

3. A. Papanestis, "A proposal for the alignment of the LHCb RICH detector", Proceedings 7th International Conference on Advanced Technology and Particle Physics, Como, October 2001, to be published by World Scientific.

4. E. Albrecht et al., Nucl. Instrum. Methods Phys. Res. A456 (2001) 190-205.

5. E. Albrecht et al., Nucl. Instrum. Methods Phys. Res. A456 (2001), 233-247.

6. W. Snoeys et al., Nucl. Instrum. Methods Phys. Res., A465 (2001), 176-189.

7. W. Snoeys et al., Nucl. Instrum. Methods Phys. Res., A466 (2001), 366-375.

8. E. Albrecht et al., Submitted to Nucl. Instrum. Methods Phys. Res. A; CERN-EP2001-051. 